colour contribure to the formation of fibrin, but he has not yet realised that these bodies are fragmentary parts and variously mod fied forms of the partially culoured blood.disos, hence he has described them as the independent bodies which he designates hæmatohlasts. I have taken great pains to prove that the so-called lymph-globule is in reality a disc-shaped body-a little smaller, but at the same time slightly thicker than the blood-disc, and that it consists of two varieties, to which I have given the names "primary" and "advanced," the former being a true cell of about the same diameter as the red blood-disc or slightly less, but having a greater thickness; the capsule of this cell is intimately applied to the nucleus, but can easily be demonstrated by osmosis. The advanced lymph-disc is the delicate, naked nucleus of this body; both these bodies exist in certain num. bers in the blood, in a visible state, and the latter is the body which is resolved into fibrin in the $1 y m p h$, as its derivative, the colourless disc, is in the blood. These lymph-discs are poured into the blood in large numbers at the subclavian and splenio veins, and for the most part, being colourless and already smooth, enter at once upon the stage of invisibility; but those among them which are not so perfectly elaborated remain for a time visible, and are at this stage colourless, finely granular discs, slightly smaller than the red discs. This appears to me to be the body to which Bizzorero refers, and as it forms fibrin while still in the lymph, so $a$ fortiori when present as one of the elements of the "fugitive group" in the blood, it is a fibrin-forming corpuscle; but the difference between the fihrin formed by this budy and the invisible and intermediate discs is precisely the difference between the coagulation of the lymph and of the blood-the one yielding, soft and granular, the other more homogeneous, viscous, and tenacious fibrin. These bodies, for many years familiar to me, must have been seen by most observers of the blood, but regarded as delicate minute forms of the white blood-corpuscle. It would indeed be a matter of surprise if they could not be seen in the circulating blood when carefully looked for, and therefore the fact' that "they are visible with their typical characteristics in their most normal condition, in the living circulating blood itself," is not the slightest disproof of the' view that they are transition forms. They are, in fact, a slightly less developed stage of the invisible colourless disc which eventuates in the red disc, and their gradual transformation into this body may be traced both in the lymph and in the blood. I have alreary incidentally referred to the allegation of Professor Bizzozero, that the invisible corpuscles of the blood as displayed by my methods are decolourised red discs. Such a view naturally occurs as a possible contingency to the mind of everyone, and it was the first point to the clearing up of which I seriously applied myself. Professor Bizzozero will pardon me for saying that he is at present only on the threshold of this research, and occupies to-day the position in which I found myself in the latter part of the year 1877. He seems to be unacquainted with my paper, published in the Proceedings of the Birmingham Philosophical Society in June, 1880, in which I deal exhaustively with this aspect of the question, and bring together numerous overwhelming proofs that this facile explanation is altogether untenable.

The singular notion that blood-discs can discharge the whole of their hæmoglobin, and become suddenly (within ten seconds) colourless not only to the eye, but to infinitely more delicate tests, of a photo-chemical nature; and that in a serum, which must (if the corpuscles lose it) necessarily contain hranglobin, or, as is the case, in a serum which has been purposely saturated with hæmoglobin (derived from an independent source) at the moment it leaves the vessels, is one entirely of modern growth-in fact, has been invented to meet my discovery. If to draw a drop of blood from the end of the finger, and to place it on a slide, either at the temperature of the room, or at that of blood or ice, and to lay down upon it in the gentlest manner a flexible mica-cover, is a manipulation calculated to bleach to whiteness some corpuscles, while leaving others and contiguous ones, under precisely the same conditions, absolutely unmodified, then certainly there is an end to all such processes as corpuscle enumeration with its diluents, measuring tubes, cells, \&c. That able investigator of the blood, Professor Gulliver, who I believe was the first to inquire into the question of the decolourisation of red discs, favoured me in 1879 with a letter referring to my first paper, in which he says, "It gives me pleasure to see that you support my original view as to the essential difference between lymph-corpuscles and the pale globules of the blood (in reality the very point now again raised from the blood side by Professor Bizzozero) ; your new corpuscle bears a great resemblance to the colourless basis of the red corpuscles. I described and figured the "membranous bases, 'and taught how to obtain them, upwards of a quarter of a century since, but this does not affect your discovery of the free and hardly seen corpuscles in the living blood; indeed if confirmed, your observation will have im. portaut signıficance." Clearly Professor Gulliver does not entertain the idea that corpuscles can be reduced to the stroma-form by the mere arrangements necessary to place blood under the microscope for examination. I might retort upon Professor Bizzozero that to examine blood-corpuscles in a solution of chloral or chloroform (for this is what happens with chloralised animals) is not nearly so normal a coudition as to examine them within a few seconds of being shed, from the end of one's own finger, in their normal undrugged plasma. Such objections raised to the simplest methods while conclusions are accepted without hesitation, when it is known that the blood contains foreign substances, capable of maintaining an animal in a profoundly narcotised condition for hours, seem to me, to say the least, "straining at a gnat and swallowing a camel." Further, I have ascertained that it is not the three-quarter per cent. saline solution alone which prevents decolourisation of the corpuscles, but the colloid matter which the blood contains, and $I$ find that by increasing this element, by adding a little dry soluble albumen to the blood, I can prevent exosmose of colouring matter from the corpuscles to such an extent that no change in them, or in the liquor sanguinis, is obvious after a period of eighteen bours. The invisible corpuscles are present as usual. Where, I ask, is the colour from these corpuscles gone to on the hypothesis that they are decolourised discs? It is not present in the liquor sanguinis, fur this photographs as clear and colourless as the adjacent air-bubbles. Numerous other reasons are given in my paper, each one of which, even when taken by itself, is decisive on this question. But to return to the main issue, what is to be done with the cardinal and indisputable fact that these identical corpuscles can be traced step by step into the well-known fibrin forms? In conclusion, I would respectfully ask Professor Bizzozero, and physiologists generally, to suspend judgment for a short period till my work on the "Physiology and Pathology of the Blood," now on the verge of publication, is issued, as it contains the evidence which I have accumulated upon these questions during the last few years.

Birmingham.

\section{CASE OF TRAUMATIC TETANUS ; RECOVERY.}

\section{BY JAMES MILLAR, M.B., C.M. ABERDEEN.}

D. $\mathrm{K}-$, aged forty-three, farm servant, a strong, healthy man, about 9 A.M. on Saturday, October 22nd, while engaged at a threshing mill, was dragged down on his side and shoulder between the wheels, which continued to revolve, and in this position he remained from three to five minutes. Being about six miles from town, an hour elapsed from the time of the accident till the patient was seen, when he was suffering slightly from shock, but bad partially recovered. When his clothes were removed it was found that he had contused and lacerated wounds of the left side and shoulder; extending from the angle of the eighth rib to the inferior angle of the scapula were five transverse wounds about two inches in length (an inch on each side of the angle of the rib) and of considerable depth. The amount of skin between them at their widest part was not more than a quarter of an inch, and this was more or liss torn. On the dorsum of the scapula, reaching to the spine of the scapula ahove, and extending to the posterior axillary border externally, was a large circular wound of irregular outline, about four inches in diameter. This wound was deepest close under the spine of the scapula, where only a few fibres of the infra-spinatus muscle covered the bone. The wound at its most superficial part reached the same muscle. This wound was filled with chopped flesh, mixed with part of the clothing. Above the spine of the scapula was another wound about two inches and a half in diameter ; this one was not so deep as the former, 
but resembled it in appearance, and over the clavicle was an abrasion of the skin ahout an inch in diameter. At the middle of the arm on the inner aspect there was a considerable contusion and a slight abrasion of the skin, embracing about half the circumference of the arm. This was the only place where the patient complained of pain, where he said it was considerable. No bones were broken. There was no bleeding except from the abraded surface over the clavicle, where there was a little oozing. The wounds were cleared of all débris as far as possible, washed out and dressed with carbolic lotion ( 1 to 40 ). The patient was laid on his right side, the left arm bandaged to the side, and the left forearm raised on a pillow in front of the patient at right angles to the body.

On the morning of the 23rd the wounds were again examined and dressed; parts of the disorganised tissue which had not been cut away the previous day were beginning to slough. A piece of oiled silk was placed over the carbolic dressing, and the whole secured by a broad bandage. The patient expressed himself as being quite comfortable, with the exception of the contusion on the arm, which was still very painful at times, but there was little discolouration of the skin round about. Temperature $99^{\circ}$; pulse quite normal. Appetite good, and the patient had slept for short periods during the previous night. The wounds were dressed twice a day, and such orders given for proper hygienic arrangements as could be carried out in a farm-servant's house. On the 24th the patient felt quite well; he had a good night. Face slightly flushed. Pulse 80 ; temperature $100^{\circ}$, and slight swelling of the forearm. The bowels had not been moved since before the accident; a dose of castor oil was ordered The bridges of skin over the angles of the ribs were now beginning to slough, and at the bottom of the wounds in the same place larue sloughs could be seen. On the 25 th the patient was still doing well; he had slept well the previous night, and the bowels had been freely moved. Temperature $99^{\circ}$; pulse 80 ; appetite good. As the discharge was becoming profuse, the wounds were dressed three times a day, and marine lint placed round the edges of the dressings. Some of the sloughs were beginning to separate; patient still complaining of the contusion on the arm. On the 26th the pulse and temperature were normal ; sloughs separating rapidly. The bowels not having been moved since the 24 th another dose of castor oil was ordered. On the 27 th he was still doing well; all the sloughs had separated, the wounds looking healthy, and some of them filling up rapidly. On the 29 th the wounds were filling up with healthy granulations, and on the 3lst were still clean and healthy.

On Nov. 2nd the wounds were beginning to contract, but the lower ones, over the angles of the ribs, had assumed a somewhat fungoid character. The patient was complaining slightly of a sore-throat, but only in the act of swallowing. On the 5th the neck was red, and painful when touched; nothing abnormal could be seen about the wounds. On the 8 th he was not very well; a poultice had been applied to the throat, but without any beneficial effect. Temperature normal. Appetite good, but slight difficulty in chewing. Pain in the throat on swallowing. Patient said this pain proceeded from the shoulder to the throat; he felt it running up to the throat. He was also complaining of what he called "grips" in the shoulder. There being an epidemic of erysipelas in the district at the time, the wounds were carefully examined, but nothing abnormal could be seen. This was about 4 P.M. At 1 A.M. the following morning he was in great agony; a teaspoon could not be inserted between the teeth, but he could swallow any fluid put into the mouth. He was bathed in perspiration, with severe tetanic contractions of the muscles of the chest, neck, and back. The body generally was much arched back. He could not open the mouth in the least, though a small space remained between the teeth in the upper and lower jaws. Risus sardonicus very well marked, and expression of great agony and exhaustion. Pulse slow and full; temperature normal. The least noise or disturbance increased the severity of the contractions. The patient had one-sixth of a grain of morphia injected deep into the gluteal muscles, and in a very few minutes the severity of the convulsions was considerably lessened, and he expressed himself as being greatly relieved; still the contractions returned as often, but he did not feel them so much. In a few minutes after one dracbm of syrup of chloral (B.P.) was administered by the mouth, the patient was still further relieved, the contractions being still as frequent, but much less severe, and, as he said himself, he could bear them. Instruc tions were given that he was to have a drachm of syrup of chloral every hour while awake. After taking three drachms of syrup of chloral he slept for about two hours when he awoke the chloral was again admivistered, and he again slept for abont an hour. On the afternoon of the 9th he was considerably under the influence of chloral. The paroxysms were not so regular in their occurrence and less severe. The contractions continued when he was asleep, but seldom awoke him, but he always had a severe one on waking. Temperature $99^{\circ}$; tongue white and furred, red at edges; the mouth could be opened a little wider. Up to the evening of the 9th he had taken one drachm of syrup of chloral every hour, except during the three hours he had been asleep, and the paroxysms were now milder, and he could bear them for some little time. He could not bear the body to be touched, and the head was still pulled back during the contractions, but not when asleep. Urine less in quantity, and dark coloured; patient not perspiring so much. On being asked to put out his tongue, the tip could not be protruded, but the dorsum, arched, was shown between the teeth, pressed hard on the teeth of the upper jaw with a jerking motion, and generally with a moist crackling sound. The patient was now ordered to take a drachm of syrup of chloral occasionally. On the 10th he was a little worse than on the previous day, and the chloral (same quantity) was given every hour. The appetite still remained good, but solid food could not be taken; the bowels were easily moved by half an ounce of castor oil every third day. In order to remove every possible source of irritation, the carbolic dressing was discontinued; the wounds, now level, and a little fungoid at the lower part, were bathed with lukewarm water, and water dressing applied. Whenever the dressings were being removed, severe paroxysms set in ; but the wounds themselves, however, could be touched, batbed, and dried with impunity, and without the slightest feeling of pain. On the 11th he was a good deal better; the contractions had extended to the abdomen and thighs, but were much less severe; he had slept fairly well the previous night, and when the contractions awoke him he had another dose of chloral. His food cnnsisted chiefly of beef-tea, hare-soup, and chicken-soup. The mouth could be opened a little wider, but the tongue could not be put outside the teeth. Another symptom observed was that the contrautions were less severe if the bandage retaining the dressings was put on loosely. He now began to take his chloral only when the contractions became severe; they now recurred regularly, but with less intensity, every twenty minutes or half hour. The ex. pression was much improved, but the risus sardonicus was still well marked. The patient went on improving till the 16th, when he was able to sit up in bed, with the body well bent forward. When he assumed this position, the contractions recurred less frequently. He found the contrac. tions were a great deal worse after dinner. He could now take a little solid food, and open the mouth pretty wide; but he would on no account be without his chloral, from which he experienced immediate relief whenever he took it. The dose of chloral syrup was now increased to $a \mathrm{drachm}$ and a half, to be taken when necessary. On the 2lst, the wounds were dressed with sulphate of zinc lotion (two grains to the ounce) and in a few days assumed a healthy character. The patient was now allowed to get up for a short time every day to sit in a chair; and in two or three days he was able to walk a little with assistance. The chloral was now entirely discontinued, and the patient went on favourably till Dec. 11 th, when he was perfectly well, not the least symptom of tetanic contraction remained, and the wounds had nearly healed.

Remarks.-As to the cause of tetanus in this case, no explanation can be offered, except that for two or three days before the onset there was dampness of the walls of the room in which he was lying. The onset and course of the symtoms were closely observed, and differ somewhat from those described in text-books-in fact, few text-books agree in the precise desciption of the symptoms. There is nothing peculiar to record about the present case, but that it had been treated successfully by chloral in a country practice; and it was considered worth recording in the pages of THE LANCET, as the simple treatment adopted can be followed by any country practitioner who has not at his disposal, nor the opportunity of watching the action of, such remedies as curara, cannabis indica, \&c., which have been used of late.

Hill-terrace, Arbroath. 